References:

[1] Tsokos GC et al. New insights into the immunopathogenesis of systemic lupus erythematosus. Nat Rev Rheumatol. 2016 Nov 22;12(12):716-30.

[2] Marinho A et al. Vitamin D supplementation effects on FoxP3 expression in T cells and FoxP3+/IL-17A ratio and clinical course in systemic lupus erythematosus patients: a study in a Portuguese cohort. Immunol Res. 2017 Feb 16;65(1):197-206.

[3] Dias AM et al. Metabolic control of $\mathrm{T}$ cell immune response through glycans in inflammatory bowel disease. Proc Natl Acad Sci U S A. 2018 May 15;115(20):E4651-60.

[4] Pereira MS \& Alves I. et al. Glycans as Key Checkpoints of T Cell Activity and Function. Front Immunol. 2018 Nov 27;9:2754.

[5] Pinho SS, Reis CA. Glycosylation in cancer: mechanisms and clinical implications. Nat Rev Cancer. 2015 Sep 20;15(9):540-55.

[6] Verhelst X et al. Protein Glycosylation as a Diagnostic and Prognostic Marker of Chronic Inflammatory Gastrointestinal and Liver Diseases. Gastroenterology. 2020 Jan 1;158(1):95-110.

Acknowledgments: S.S.P. and I.A. acknowledge Group of Studies for Autoimmune diseases (NEDAI) from Portuguese Society of Internal Medicine (SPMI) for Funding and for supporting the attendance at this meeting.

Disclosure of Interests: None declared

DOI: 10.1136/annrheumdis-2020-eular.1231

\section{AB0124 NO EFFECTS OF HIGH DOSE 25OH-VITAMIN D SUPPLEMENTATION ON LUPUS NEPHRITIS IN AN ANIMAL MODEL OF SYSTEMIC LUPUS ERYTHEMATOSUS}

S. Barsotti ${ }^{1}$, C. Tani ${ }^{1}$, A. Kuhl' ${ }^{2}$, S. Pacini ${ }^{3}$, S. Vagnani ${ }^{1}$, M. Mosca' ${ }^{1}$.

${ }^{1}$ Rheumatology Unit - Pisa University Hospital, Pisa, Italy; ${ }^{2}$ Charité -

Universitätsmedizin Berlin, corporate member of Freie Universität Berlin, Humboldt Universität zu Berlin, and Berlin Institute of Health, iPATH.Berlin, Germany, Berlin, Germany; ${ }^{3}$ Haematology Unit - Pisa University Hospital, Pisa, Italy

Background: $25 \mathrm{OH}$ Vitamin $\mathrm{D}$ (25-OH-D3) is a fat-soluble steroid-derived molecule involved in the calcium homeostasis. Low levels of 25-OH-D3 are commonly found in patients with systemic lupus erythematosus (SLE) and have been correlated to higher disease activity and severity. Several recent studies have demonstrated that high dose Vitamin D may influence several aspects of the innate and adaptive immune response and some authors hypothesized that high dose 25-OH-D3 may have a role in the treatment of SLE. Despite these observations, the immunomodulatory effect of high dose $25-\mathrm{OH}-\mathrm{D} 3$ in vivo still needs to be demonstrated.

Objectives: The aim of our study was to identify the effect of 25-OH-D3 on proteinuria, survival and renal biopsy in New Zealand Black/White F1 mice (NZ), that develop a disease very similar to human SLE nephritis.

Methods: We administered to $20 \mathrm{NZ}$ mice a diet enriched with high dose $25-\mathrm{OH}-\mathrm{D} 3 \mathrm{10}, 000 \mathrm{UI} / \mathrm{Kg}$ starting from 8 weeks of age. Mice were divided in 7 experimental groups ( 5 mice each). The first group was sacrificed before the start of the treatment ( 8 week of age), three groups were treated (treated mice - TM) with 25-OH-D3 and sacrificed at 16,26 and 36 weeks of age. The other three groups were enrolled as controls and sacrificed at 16, 26 and 36 weeks of age respectively (untreated mice - UM). The parameters collected included: total urinary protein and kidney histology for the evaluation of lupus nephritis (LN): glomerulonephritis, interstitial nephritis and vascular lesions according to a 5 points scale to obtain a total score (ranging from 0 to 12).

Results: In UM, proteinuria tended to increase over $1 \mathrm{mg} /$ day at 12 weeks of age $(1.7 \pm 0.43 \mathrm{mg} /$ day $)$ and further increased until to reach a plateau after 28 weeks of age $(10 \pm 2.0 \mathrm{mg} /$ day $)$

In TM, a significant increase in proteinuria over $1 \mathrm{mg} /$ day was observed at 24 weeks, when the mean proteinuria was $1.7 \pm 1.33$ which was lower than controls at the same age although without statistical significance $(2.9 \pm 2.6)$; thereafter proteinuria started to increase also in treated mice and at week 30 was higher in TM compared with UM $(10,3 \pm 8.8$ vs $4.3 \pm 3.5 p=0.05)$. Figure 1 . Kidney histology showed, in mice sacrificed before the start of the treatment no signs of LN. In mice sacrificed at 16 weeks minimal interstitial nephritis (score 1) was identified in 2 mice only in UM. At 26 weeks of age, a higher total LN score was identified in TM compared with UM $(3.4 \pm 3.8$ vs $0.4 \pm 0.9)$ with higher score for all three parameters analyzed. At 36 weeks of age, the TM group maintained a higher total LN score compared to UM $(6.5 \pm 1.7$ vs $6.0 \pm 2.6)$ with higher score for glomerulonephritis and interstitial nephritis.
In the TM group, three mice spontaneously died at 26,30 and 32 weeks of age, while in the UM only one mouse died at 36 weeks of age.

Conclusion: Our data suggest that, in this animal model of SLE, 25-OH-D3 administration seems to delay the onset of proteinuria, although has no effect on the overall disease control. In addition, it may have a negative effect on renal histology and survival with earlier development of LN.

Figure:

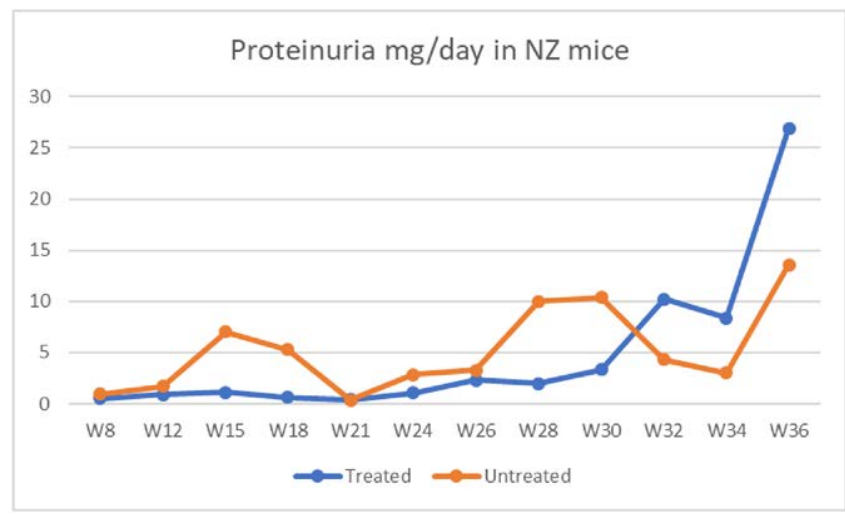

Disclosure of Interests: None declared DOI: 10.1136/annrheumdis-2020-eular.4250

\begin{tabular}{|l|l}
\hline AB0125 & EXPRESSION OF INTERFERON TYPE I- AND TYPE \\
II-INDUCED GENES IN PATIENTS WITH SJÖGREN'S \\
SYNDROME WITH AND WITHOUT EXTRAGLANDULAR \\
INVOLVEMENT
\end{tabular}

N. Del Papa ${ }^{1}$, C. Vitali ${ }^{2}$, M. Lorini ${ }^{3}$, V. Carbonelli ${ }^{3}$, W. Maglione ${ }^{1}$, F. Pignataro ${ }^{1}$, A. Minniti ${ }^{1}$, N. Montano ${ }^{3}$, R. Caporali ${ }^{1} .{ }^{1}$ ASST Gaetano Pini-CTO, Dept of Rheumatology, Milan, Italy; ${ }^{2}$ Mater Domini Humanitas Hospital, Castellanza, Italy; ${ }^{3} \mathrm{Ca}$ ' Granda IRCCS Foundation, Ospedale Maggiore Policlinico, Department of Clinical Sciences and Community Health, Milan, Italy

Background: It is well known that Sjögren's syndrome $(\mathrm{SjS})$ is characterized by an upregulation of interferon (IFN)-induced genes. Namely, IFN type I signature has been reported in peripheral blood mononuclear cells (PBMCs) and in salivary glands of patients with this disease. However, few data are available on possible variability of IFN-induced gene upregulation in different clinical phenotypes of SjS.

Objectives: To verify whether upregulation of IFN-induced genes is comparable in patients with $\mathrm{SjS}$ characterized by different clinical phenotypes, i.e. patients with systemic extraglandular manifestations (EGMs) versus patients with a disease limited to glandular features (GFs) and with widespread pain (WP).

Methods: The study population was composed by 11 patients with SjS and EGMs (1 male, age range 18-78 years), and 10 patients with only GFs and WP (all females, age range 46-81 years), all classified according to ACR-EULAR criteria. The prevalence of anti-SSA(Ro) antibodies was $11 / 11$ and $8 / 10$, respectively. Lip biopsy was positive in all cases. Six healthy normal subjects were also included in the study as control population.

Four IFN type I- and 5 IFN type II-induced genes were chosen for the study on the basis of previous literature data. Total RNA from each patient and control was isolated from purified PBMCs, followed by CDNA preparation and real time quantitative-PCR (RQ-PCR) analysis, using specific primer/probe sets. For calculation of relative expression, all samples were normalised against expression of a household gene (beta actin). A further normalization was performed against the mean value of relative expression obtained in the normal controls. Final fold change values were determined from the double-normalised values using the 2- $\Delta \Delta \mathrm{CT}$ method (Applied Biosystems).

Results: Fold change values of gene expression of both IFN type I- and type II-induced genes in PBMCs were different in the two clinical phenotypes of SjS. Fold change values of IFN type I-induced genes appeared strongly higher in patients with EGM, and some of them only moderately increased in those with only GF and WP. The expression of some of IFN type Il-induced genes were slightly increased in patients belonging to both clinical phenotypes. Results are detailed in the table. 
Table. Fold change values of gene expression in patients with SjS plus EGMs, in patients with disease limited to GF and WP, and in controls.

\begin{tabular}{lrrrrrrrrr}
\hline Gene & MX1 & IFIT1 & IFT3 & IFI44 & IDO1 & GRP1 & MIG & IP-10 & P2RY14 \\
\hline SjS-EGMs & 85.9 & 38.5 & 24.4 & 40.4 & 25.1 & 8.3 & 4.5 & 1.5 & 5.5 \\
SJS-GF-WP & 4.2 & 1.7 & 2.0 & 4.8 & 4.1 & 1.2 & 0.6 & 0.3 & 1.3 \\
Controls & 2.1 & 1.6 & 1.1 & 1.5 & 1.4 & 1.3 & 1.5 & 1.3 & 1.4
\end{tabular}

Legend. IFN type I-induced genes: MIX, IFN-induced GTP binding protein 1; IFIT1, IFNinduced protein with tetratricopeptide repeats 1 ; IFIT3, IFN-induced protein with tetratricopeptide repeats 3; IFI44, IFN-induced protein 44

IFN type II-induced genes: IDO1, indolamine-deoxygenase 1; GBP1, guanylate binding protein 1; MIG, C-X-C chemokine 9 (CXCL9); IP-10, C-X-C chemokine 10 (CXCL10); P2RY14, purinergic receptor 14

Conclusion: The present data indicate that IFN type I- and, to a lesser degree, type II-induced genes are upregulated in patients with SjS, but this phenomenon is consistently stronger in patients with systemic EGMs. In patients with only GFs IFN-induced gene upregulation is milder in PBMCs, and then probably more restricted to the exocrine target tissues.

Disclosure of Interests: Nicoletta Del Papa: None declared, Claudio Vitali: None declared, Maurizio Lorini: None declared, Vincenzo Carbonelli: None declared, Wanda Maglione: None declared, Francesca Pignataro: None declared, Antonina Minniti: None declared, Nicola Montano: None declared, Roberto Caporali Consultant of: AbbVie; Gilead Sciences, Inc.; Lilly; Merck Sharp \& Dohme; Celgene; Bristol-Myers Squibb; Pfizer; UCB, Speakers bureau: Abbvie; Bristol-Myers Squibb; Celgene; Lilly; Gilead Sciences, Inc; MSD; Pfizer; Roche; UCB

DOI: 10.1136/annrheumdis-2020-eular.2845

\begin{tabular}{|l|l}
\hline AB0126 & EXPRESSION CHARACTERISTICS OF ADENOSINE \\
DEAMINASES ACTING ON RNA-1 IN SYSTEMIC LUPUS \\
ERYTHEMATOSUS AND ITS CORRELATION WITH \\
SERUM IFN-A
\end{tabular}

X. Di ${ }^{1}$, F. Gao ${ }^{2}$, C. Gao ${ }^{1}$, C. Zhang ${ }^{1}$, W. Sun ${ }^{1}$, W. Liang ${ }^{1}$, M. Yao ${ }^{1}$, Q. Wang ${ }^{1}$, Z. Zheng ${ }^{1}{ }^{1}$ The First Affiliated Hospital of Zhengzhou University, Zhengzhou, China; ${ }^{2}$ Zhengzhou University, Zhengzhou, China

Background: SLE is a multisystem autoimmune disease characterized by the production of multiple autoantibodies and loss of immunity against autoantigens in various tissues. SLE patients have significantly elevated RNA editing levels and the potential to produce new autoantigens. ${ }^{1}$ ADAR1 is an RNA A-I editing enzyme that converts adenine to hypoxanthine and contributes to SLE pathogenesis. $^{2}$

Objectives: Dama demonstrated the upregulation of ADAR1p150 expression in SLE T cells, B cells, PBMCs, and NK cells, ${ }^{3}$ however, the following issues were not reported in detail: 1. specific alterations in ADAR1 expression in PBMCs collected from SLE patients with varying degrees of the disease and its correlation with serum IFN-a levels; 2. association between ADAR1 and clinical indicators; and 3. ADAR1 expression in renal tissue of LN patients. Our study therefore aimed to elucidate the abovementioned points.

Methods: We used qRT-PCR to determine ADAR1 expression levels in PBMCs and renal tissues of controls and SLE patients. We also conducted immunohistochemical studies to detect positive ADAR1 expression rate in renal cells of controls and LN patients.

Results: ADAR1 expression was higher in PBMCs of SLE patients than in those of controls and was positively correlated with SLEDAI. When serum IFN-a levels in SLE patients decreased $<260.0 \mathrm{pg} / \mathrm{mL}$, ADAR1 expression in PBMCs increased with the increase in IFN- $a$ concentration, and serum IFN- $a$ may regulate ADAR1 level in PBMC in SLE patients, which may require the participation of serum IgG antibody and related immune complex. However, there was no significant difference between the expression in renal tissues in all patients.

Conclusion: There was a certain correlation between ADAR1 expression and serum IFN-a levels in PBMCs of SLE patients.

References:

[1] Roth SH, Danan-Gotthold M, Ben-Izhak M, et al. Increased RNA Editing May Provide a Source for Autoantigens in Systemic Lupus Erythematosus. Cell Rep 2018; 23: 50-57.

[2] Hogg M, Paro S, Keegan LP and O'Connell MA. RNA editing by mammalian ADARs. Adv Genet 2011; 73: 87-120.

[3] Laxminarayana D, Khan IU, O'Rourke KS and Giri B. Induction of 150-kDa adenosine deaminase that acts on RNA (ADAR)-1 gene expression in normal T lymphocytes by anti-CD3-epsilon and anti-CD28. Immunology 2007; 122: 623-633.
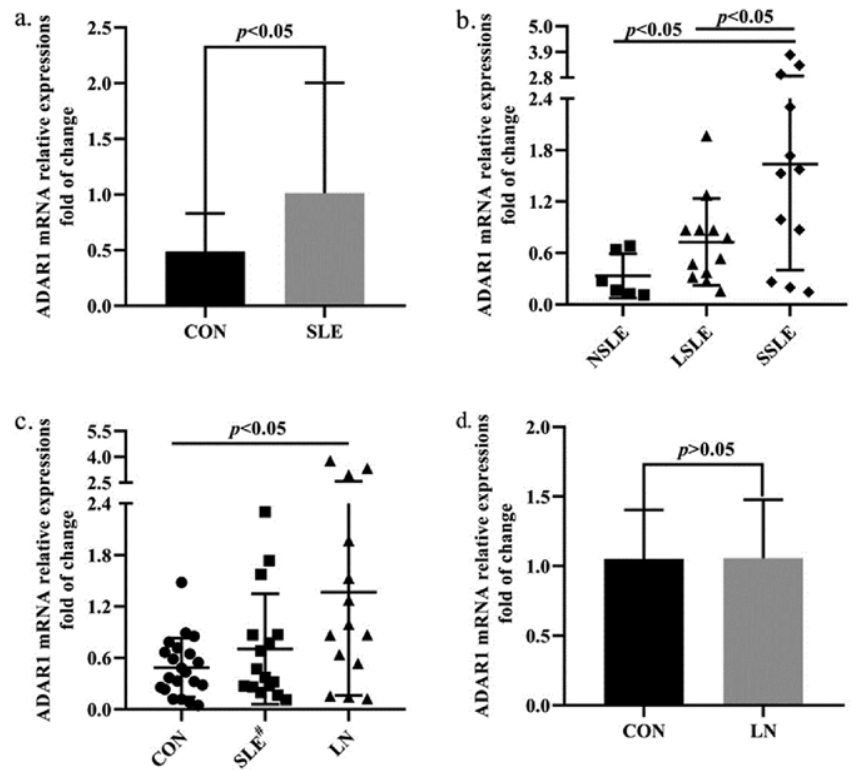

Figure 1. Analysis of ADAR1 expression levels. a. The ADAR1 expression in PBMCs was higher in SLE patients $(n=30)$ than in healthy controls $(n=30)(p<0.05)$. b. SLE patients were divided into three groups: NSLE (SLEDAI 0-4, $n=6$ ), LSLE (SLEDAI 5-9, $n=12$ ), and SSLE (SLEDAI $\geq 10, n=12$ ) according to SLEDAI score. $c$. Based on the effect of the disease on the kidneys, the patients were divided into the SLE ${ }^{\#}$ group (\#:SLE patient group without the kidney involved, $n=17$ ) and LN group (lupus nephritis group, $n=13$ ). d. There was no significant difference observed between the renal tissues of controls $(n=5)$ and LN patients $(\mathrm{n}=10)(p>0.05)$

a.

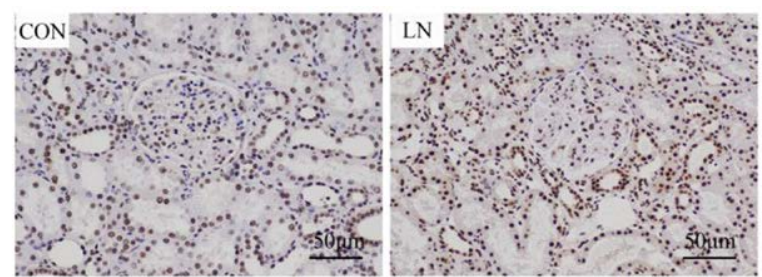

b.
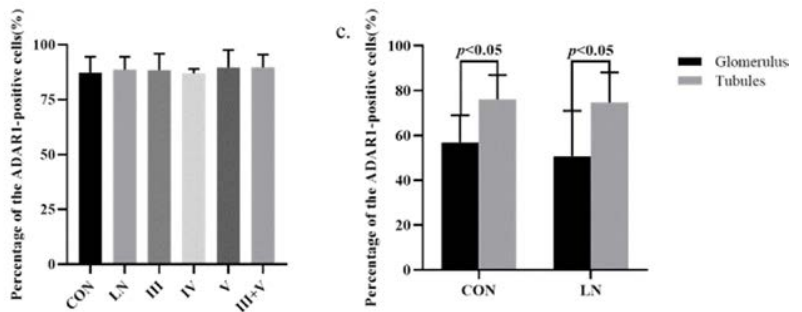

Figure 2. a. Immunohistochemical image of renal tissues from the two groups (200x). b. There was no significant difference in the ADAR1 cell positive rate between controls $(n=5)$, LN patients $(n=20)$, and different pathological subgroups (class III, $n=5$; class IV, $n=5$; class $V$ $\mathrm{n}=5$; class III+IV, $\mathrm{n}=5)(p>0.05)$. c. The positive expression rate of ADAR1 in renal tubular cells was higher than that in glomerular cells both in the two groups $(p<0.05)$.

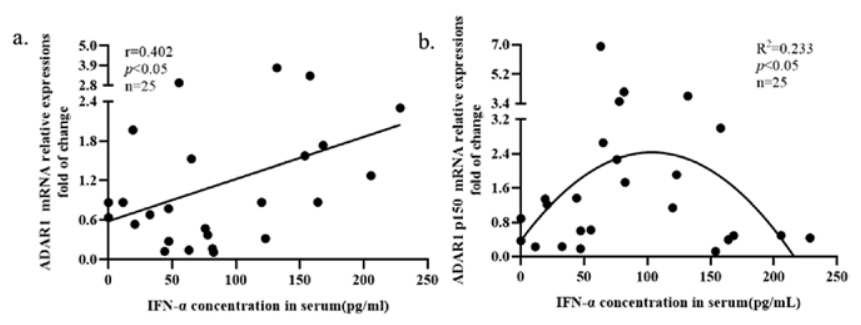

Figure 3. a. Correlation between ADAR1 and serum IFN- $\alpha$ levels in PBMCs of SLE patients. b. Correlation between ADAR1p150 and serum IFN-a levels in PBMCs of SLE patients. 